\title{
Structure of III-Sb(001) Growth Surfaces: The Role of Heterodimers
}

\section{Citation}

Barvosa-Carter, W., A. S. Bracker, J. C. Culbertson, B. Z. Nosho, B. V. Shanabrook, L. J. Whitman, Hanchul Kim, N. A. Modine, and E. Kaxiras. 2000. "Structure of III-Sb(001) Growth Surfaces: The Role of Heterodimers." Physical Review Letters 84 (20): 4649-52. https:// doi.org/10.1103/physrevlett.84.4649.

\section{Permanent link}

http://nrs.harvard.edu/urn-3:HUL.InstRepos:41384111

\section{Terms of Use}

This article was downloaded from Harvard University's DASH repository, and is made available under the terms and conditions applicable to Other Posted Material, as set forth at http:// nrs.harvard.edu/urn-3:HUL.InstRepos:dash.current.terms-of-use\#LAA

\section{Share Your Story}

The Harvard community has made this article openly available. Please share how this access benefits you. Submit a story.

Accessibility 


\title{
Structure of III-Sb(001) Growth Surfaces: The Role of Heterodimers
}

\author{
W. Barvosa-Carter,* A. S. Bracker, J. C. Culbertson, B. Z. Nosho, B. V. Shanabrook, and L. J. Whitman ${ }^{\dagger}$ \\ Naval Research Laboratory, Washington, D.C. 20375 \\ Hanchul Kim, ${ }^{1}$ N. A. Modine,,${ }^{1,2}$ and E. Kaxiras ${ }^{1}$ \\ ${ }^{1}$ Harvard University, Cambridge, Massachusetts 02138 \\ ${ }^{2}$ Sandia National Laboratories, Albuquerque, New Mexico 87185
}

(Received 16 November 1999)

\begin{abstract}
We have determined the structure of AlSb and GaSb (001) surfaces prepared by molecular beam epitaxy under typical $\mathrm{Sb}$-rich device growth conditions. Within the range of flux and temperature where the diffraction pattern is nominally $(1 \times 3)$, we find that there are actually three distinct, stable $(4 \times 3)$ surface reconstructions. The three structures differ from any previously proposed for these growth conditions, with two of the reconstructions incorporating mixed III-V dimers within the Sb surface layer. These heterodimers appear to play an important role in island nucleation and growth.
\end{abstract}

PACS numbers: 68.35.Bs, 61.16.Ch, 73.61.Ey, 81.15.Hi

The surface reconstruction on a semiconducting material is the starting point for understanding the mechanisms of growth from the vapor. The steric and energetic landscape of the surface reconstruction determines the kinetic factors for adsorption, diffusion, and desorption, and provides the template for island nucleation [1]. These factors are critical to our understanding of growth and the formation of interfaces between materials, particularly for the case of III-V semiconductor quantum heterostructures, which are key components in a wide range of optical and high frequency electronic devices under development. Many of the most promising applications require extremely thin layers, so that even submonolayer variations in film thickness and interfacial roughness can dramatically affect the ultimate device performance [2,3]. To achieve the level of morphological control needed to reproducibly fabricate optimized III-V devices, a detailed understanding of the relevant surface reconstructions and the mechanisms by which epitaxy proceeds is essential.

The structure of III-As and III-P (001) surfaces under typical device growth conditions (V/III flux $>1$ ) has been generally established $[4,5]$. For the much-studied case of GaAs, the extensive experimental and theoretical knowledge accumulated about the surface structure has led to significant progress in understanding the atomistic mechanisms of growth during molecular beam epitaxy (MBE) $[6,7]$. In contrast, the III-Sb device surfaces are poorly understood, despite their demonstrated potential for a variety of advanced electronic and optoelectronic applications [8]. Although the surface structures have been determined for atypical, extreme Sb-rich conditions - InSb and AlSb have the $c(4 \times 4)$ structure common to the arsenides, and $\mathrm{GaSb}$ reconstructs into unusual, metallic $(n \times 5)$ structures $[9,10]$ - even after more than 20 years of study [11], the atomic-scale structures under more typical growth conditions have yet to be resolved. During device growth both $\mathrm{GaSb}$ and AlSb usually exhibit a $(1 \times 3)$-like reflection high-energy electron diffraction (RHEED) pattern, with the GaSb RHEED further delineated into distinct $c(2 \times 6)$ and $(1 \times 3)$ (higher temperature/lower $\mathrm{Sb}$ flux) regimes $[12,13]$. Simple Sb-dimer row models for these reconstructions have been proposed [13,14], but scanning tunneling microscopy (STM) studies of the III-Sb(001) surfaces by a number of different research groups show that the actual $(1 \times 3)$-like structures must be more complex [9,14-16].

In this Letter, we describe the atomic-scale structures that occur for a range of $\mathrm{Sb}$-rich growth conditions on $\mathrm{AlSb}$ and $\mathrm{GaSb}(001)$ as definitively determined by a combined experimental and theoretical study. We find that there are two distinct $(4 \times 3)$ reconstructions relevant for typical device growth, and that they both incorporate novel III-Sb heterodimers that appear to play an important role in island nucleation and growth. For AlSb there is a third $(4 \times 3)$-like reconstruction where the heterodimer is replaced by an $\mathrm{Sb}$ dimer, and we show using first-principles calculations that these three reconstructions are the most thermodynamically stable for AlSb, in direct agreement with experiment.

The AlSb and GaSb surfaces were prepared by MBE and characterized using STM in an interconnected, multichamber ultrahigh vacuum facility [17]. The surface reconstructions were studied on $p$-type (Be-doped, $2 \times$ $\left.10^{16} \mathrm{~cm}^{-3}\right)$, strain-relaxed films $(>1 \mu \mathrm{m}$ thick) grown at $610{ }^{\circ} \mathrm{C}$ and $520{ }^{\circ} \mathrm{C}$, respectively, on $\mathrm{GaSb}(001)$ substrates. The final 30 monolayers (ML) of each film were left undoped. RHEED was used to monitor surface order. All STM images were acquired in constant current mode using bias voltages between $-3.5 \mathrm{~V}$ (filled states) and $+2.0 \mathrm{~V}$ (empty states) and tunneling currents between 0.03 and $1.0 \mathrm{nA}$.

By varying the substrate temperature and $\mathrm{Sb}_{4}$ flux incident on the surface before quenching, we have discovered that the " $(1 \times 3)$ " phase region for AlSb is, in fact, composed of three phases that appear at successively lower temperatures and higher $\mathrm{Sb}_{4}$ fluxes. High-resolution STM images of the different structures are presented in Fig. 1. 


\section{Report Documentation Page}

Form Approved

OMB No. 0704-0188

Public reporting burden for the collection of information is estimated to average 1 hour per response, including the time for reviewing instructions, searching existing data sources, gathering and maintaining the data needed, and completing and reviewing the collection of information. Send comments regarding this burden estimate or any other aspect of this collection of information,

including suggestions for reducing this burden, to Washington Headquarters Services, Directorate for Information Operations and Reports, 1215 Jefferson Davis Highway, Suite 1204, Arlington

VA 22202-4302. Respondents should be aware that notwithstanding any other provision of law, no person shall be subject to a penalty for failing to comply with a collection of information if it

does not display a currently valid OMB control number.

\begin{tabular}{|c|c|c|}
\hline $\begin{array}{l}\text { 1. REPORT DATE } \\
\text { NOV } 1999\end{array}$ & 2. REPORT TYPE & $\begin{array}{l}\text { 3. DATES COVERED } \\
\text { 00-00-1999 to 00-00-1999 }\end{array}$ \\
\hline \multirow{3}{*}{\multicolumn{2}{|c|}{$\begin{array}{l}\text { 4tructure of III-Sb(001) Growth Surfaces: The Role of Heterodimers } \\
\text { Structime }\end{array}$}} & 5a. CONTRACT NUMBER \\
\hline & & 5b. GRANT NUMBER \\
\hline & & 5c. PROGRAM ELEMENT NUMBER \\
\hline \multirow{3}{*}{\multicolumn{2}{|c|}{ 6. AUTHOR(S) }} & 5d. PROJECT NUMBER \\
\hline & & 5e. TASK NUMBER \\
\hline & & 5f. WORK UNIT NUMBER \\
\hline \multicolumn{2}{|c|}{$\begin{array}{l}\text { 7. PERFORMING ORGANIZATION NAME(S) AND ADDRESS(ES) } \\
\text { Naval Research Laboratory, } \mathbf{4 5 5 5} \text { Overlook Avenue } \\
\text { SW,Washington,DC,20375 }\end{array}$} & $\begin{array}{l}\text { 8. PERFORMING ORGANIZATION } \\
\text { REPORT NUMBER }\end{array}$ \\
\hline \multirow{2}{*}{\multicolumn{2}{|c|}{ 9. SPONSORING/MONITORING AGENCY NAME(S) AND ADDRESS(ES) }} & 10. SPONSOR/MONITOR'S ACRONYM(S) \\
\hline & & $\begin{array}{l}\text { 11. SPONSOR/MONITOR'S REPORT } \\
\text { NUMBER(S) }\end{array}$ \\
\hline
\end{tabular}

12. DISTRIBUTION/AVAILABILITY STATEMENT

Approved for public release; distribution unlimited

13. SUPPLEMENTARY NOTES

14. ABSTRACT

15. SUBJECT TERMS

16. SECURITY CLASSIFICATION OF:

\begin{tabular}{c|c|c}
$\begin{array}{c}\text { a. REPORT } \\
\text { unclassified }\end{array}$ & $\begin{array}{c}\text { b. ABSTRACT } \\
\text { unclassified }\end{array}$ & $\begin{array}{c}\text { c. THIS PAGE } \\
\text { unclassified }\end{array}$
\end{tabular}

17. LIMITATION OF ABSTRACT

Same as

Report (SAR)
18. NUMBER 19a. NAME OF

OF PAGES RESPONSIBLE PERSON

4 


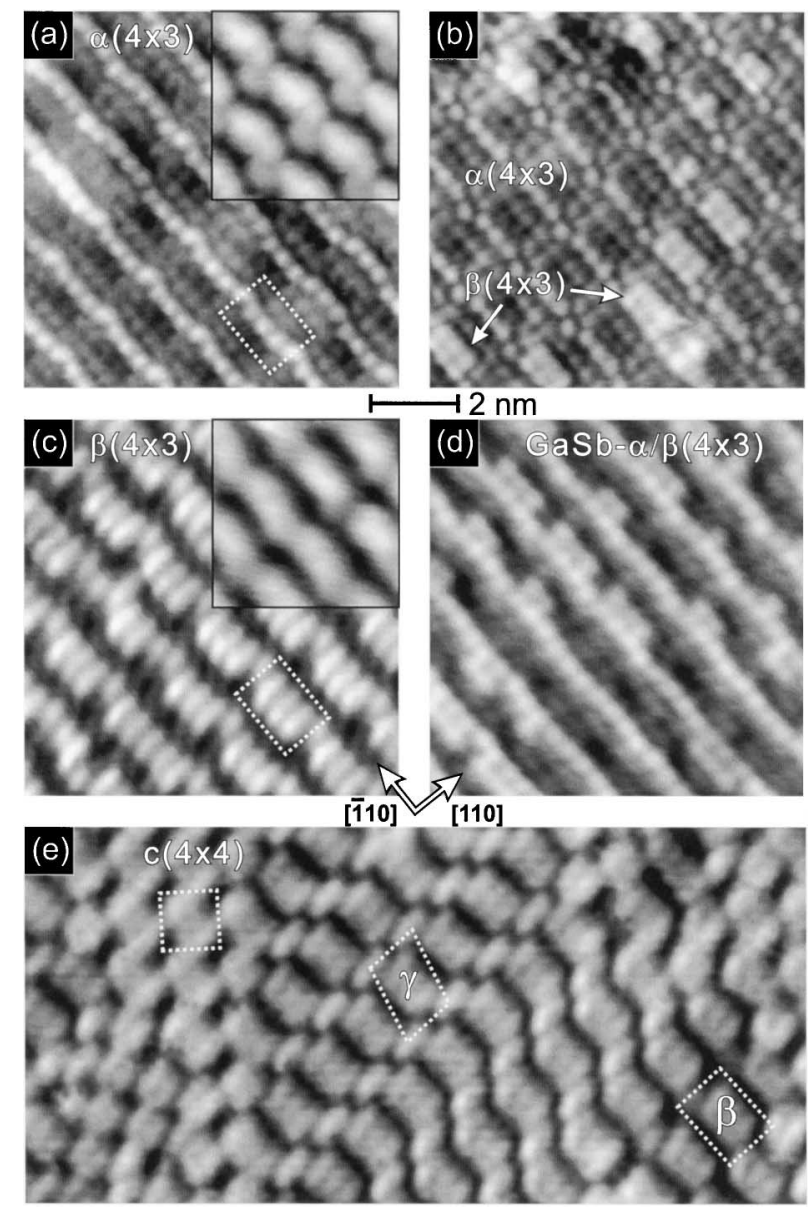

FIG. 1. (a)-(c) Filled-state STM images of the " $(1 \times 3)$ " reconstructions observed on $\mathrm{AlSb}(001)$ at constant $\mathrm{Sb}$ flux and decreasing temperature. The surface structure evolves from (a) $\alpha(4 \times 3)$, at low or no $\mathrm{Sb}$ flux, through (b) a region of mixed $\alpha$ and $\beta(4 \times 3)$, to (c) $\beta(4 \times 3)$ under typical device growth conditions. Empty-state images are shown in the insets. (d) The $\alpha$ and $\beta(4 \times 3)$ as observed on $\mathrm{GaSb}(001)$. (e) A quenched mixture of the $c(4 \times 4), \gamma(4 \times 3)$, and $\beta(4 \times 3)$ phases on AlSb under high Sb-flux/low temperature conditions. The dashed boxes outline the unit cells.

Following the nomenclature for GaAs, we denote these new phases $\alpha(4 \times 3), \beta(4 \times 3)$, and $\gamma(4 \times 3)$ in the order that they are observed under conditions of constant $\mathrm{Sb}_{4}$ flux and decreasing substrate temperature. The simplest method for forming $\alpha(4 \times 3)$ [Fig. 1(a)] is to anneal the surface near the growth temperature for several minutes without any incident flux. The $\beta$ structure, shown in Fig. 1(c), is easily stabilized by $\mathrm{Sb}_{4}$ fluxes typically used during growth. If a $\beta$ surface is annealed only briefly or under very low $\mathrm{Sb}$ flux, a mixture of $\alpha$ and $\beta$ structures are observed [Fig. 1(b)]. Curiously, although the filled-state images for $\alpha$ and $\beta$ are distinctly different, the empty-state images have a similar appearance. The $\gamma$ phase is observed only under a high $\mathrm{Sb}_{4}$ flux with the substrate held carefully near the " $(1 \times 3)$ "-to- $c(4 \times 4)$ transition temperature. $\gamma(4 \times 3)$ can be thought of as a transitional structure between the $\beta$ and $c(4 \times 4)$ phases, as illustrated in Fig. 1(e) where these three phases are all seen together. It is important to note that surfaces quenched during AlSb epitaxy under typical conditions always exhibit the $\beta$ reconstruction, even for thin films on InAs substrates [18], leading us to conclude that this is the reconstruction usually present on the surface during device growth.

RHEED typically shows a clear transition between $\operatorname{AlSb}(001)-c(4 \times 4)$ and $(1 \times 3)$ patterns as the temperature is raised. The $(4 \times 3)$ pattern of the $\gamma$ surface appears only briefly, if at all. Both $\alpha$ and $\beta$ give $(1 \times 3)$ patterns under most conditions. This observation is consistent with the STM images, which show considerable disorder with respect to the $4 \times$ periodicity. Weak row-to-row ordering of the $4 \times$ may be observed in both RHEED and STM for some surface preparation conditions. The transition from $\beta$ to $\alpha$ appears as gradual intensity changes in the $(1 \times 3)$ RHEED pattern. This gradual change suggests a second order phase transition and is consistent with the STM results, which often show intermixed $\alpha+\beta$ with no clear phase boundaries.

We find that the $\alpha$ and $\beta(4 \times 3)$ structures observed on AlSb also occur on $\mathrm{GaSb}(001)$, as demonstrated by the striking similarity between the atomic-resolution images of mixed $\alpha+\beta$ surfaces shown in Figs. 1(b) (AlSb) and $1(\mathrm{~d})(\mathrm{GaSb})$. To date we have not observed the $\gamma$ phase on $\mathrm{GaSb}$. This is perhaps not surprising given that $\gamma$ appears to be structurally intermediate between $\beta$ and $c(4 \times 4)$, and on GaSb the $c(4 \times 4)$ phase is not observed [the unique $(n \times 5)$ phases occur under the corresponding conditions]. We have preliminary results indicating that similar structures also occur on $\operatorname{InSb}(001)$. These observations lead us to suggest that the $\alpha(4 \times 3)$ and $\beta(4 \times 3)$ reconstructions are common to all the antimonides within the range of typical device growth conditions.

To determine the structure of the observed reconstructions and assess their relative stability, we have performed first-principles calculations of the ground-state geometries and surface energies of an extensive variety of possible structural models, including the previously proposed $(1 \times 3)$ and $c(2 \times 6)$ structures. The calculations were performed within the local density approximation of density functional theory using the Vienna $A b$ initio Simulation Package [19]. Each reconstructed surface was modeled on a slab of three AlSb bilayers separated by $16 \AA$ of vacuum. The $(4 \times 3)$ surface of the slab was terminated by an additional plane of Sb plus $\frac{2}{3} \mathrm{ML}$ of surface atoms in dimers, with the opposing surface terminated by fictitious fractionally charged hydrogen. Atoms were represented using ultrasoft pseudopotentials [20] as supplied by Kresse and Hafner [21]. We used a plane-wave cutoff of $11.5 \mathrm{Ry}$ and $k$-point sampling equivalent to $144 k$-points within the $(1 \times 1)$ surface Brillouin zone. All $(4 \times 3)$ surface atoms and the top two AlSb bilayers were allowed to relax until their rms forces were $<0.01 \mathrm{eV} / \AA$. Constant-current STM images 
were simulated as the height contours of an isosurface of energy-integrated local density of states.

We have confirmed that the structures with the lowest calculated surface energy are exactly those we observe experimentally by comparing the simulated constant-current STM images with the atomic-resolution experimental images. In Fig. 2 we present simulated empty and filledstate images along with structural models for each of the $\mathrm{Sb}$-terminated phases observed on $\mathrm{AlSb}(001)$. The correspondence between the experimental and theoretical images is striking - even subtle features such as the relative positions and heights of features seen in the STM images are reproduced. Furthermore, the structure with the lowest calculated surface energy as a function of increasing $\mathrm{Sb}$ chemical potential, $\mu_{\mathrm{Sb}}$, changes from $\alpha$ to $\beta$ to $\gamma(4 \times 3)$ to $c(4 \times 4)$, in direct correspondence with the experimental observations as a function of $\mathrm{Sb}$ flux. The excellent agreement between theory and experiment combined with the wide range of structures and experimental conditions explored lead us to conclude that we have identified all the stable reconstructions that occur under Sb-rich conditions on $\mathrm{AlSb}(001)$ (and probably $\mathrm{GaSb}$ and $\mathrm{InSb}$ as well).

The three $(4 \times 3)$ reconstructions are all structural permutations of the originally proposed $c(2 \times 6)$ model, which consisted of a full plane of $\mathrm{Sb}$ atoms covered
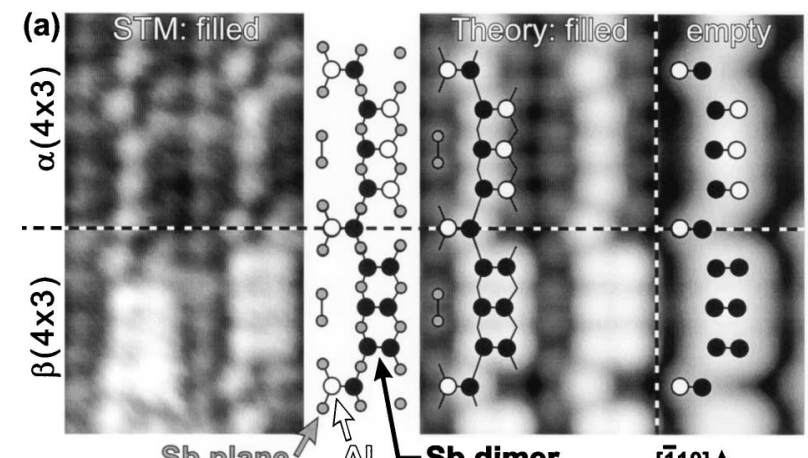

(b)

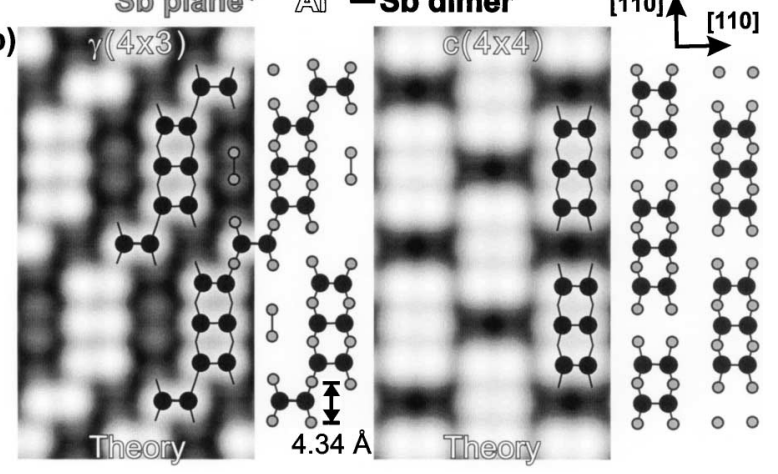

FIG. 2. Structural models and atomic-scale constant-current images of the stable Sb-rich $\mathrm{AlSb}(001)$ reconstructions. (a) A comparison of the experimental (left) and simulated (right) filled-state STM images for $\alpha(4 \times 3)$ (top) and $\beta(4 \times 3)$ (bottom). Simulated empty-state images are also shown on the right [compare with the insets in Figs. 1(a) and 1(c)]. (b) Simulated images of the $\gamma(4 \times 3)$ and $c(4 \times 4)$ phases [compare with Fig. 1(e)]. by $\frac{2}{3} \mathrm{ML}$ of $\mathrm{Sb}$ in surface dimer rows, each separated by a trench containing rotated dimers in the full plane below. All the $(4 \times 3)$ structures involve the addition of a kink every fourth dimer that moves the dimer by one lattice constant in the [110] direction. Formation of this kink creates two half-filled dangling bonds in the trench that can accept and precisely compensate for the extra electrons that otherwise would cause the $c(2 \times 6)$ to violate the "electron counting" model. Although we have not performed tunneling spectroscopy on the $(4 \times 3)$ surfaces of AlSb, based on the calculated band structures, which all exhibit a gap, we would expect them to be semiconducting like the $c(4 \times 4)$ reconstruction [10].

If the $\mathrm{Sb}$ flux is high enough, no Al-for-Sb exchange occurs, and the $\gamma(4 \times 3)$ surface results $\left(1 \frac{2}{3}\right.$ ML surface $\mathrm{Sb}$; see Fig. 2). However, as the $\mathrm{Sb}$ flux is reduced, corresponding to a decrease in $\mu_{\mathrm{Sb}}$, the energy is lowered by aligning the $\mathrm{Sb}$ dimer rows and replacing one $\mathrm{Sb}$ atom in each kink dimer with an $\mathrm{Al}$ atom (always the atom between the rows). The resulting $\beta$ reconstruction is terminated by $1 \frac{7}{12} \mathrm{ML} \mathrm{Sb}+\frac{1}{12} \mathrm{ML}$ Al. With further decrease in $\mu_{\mathrm{Sb}}$, this substitution occurs in the other three top-row dimers and $\alpha(4 \times 3)$ is formed with $1 \frac{1}{3} \mathrm{ML} \mathrm{Sb}+\frac{1}{3} \mathrm{ML} \mathrm{Al}$. Note that this progressive substitution of $\mathrm{Al}$ for $\mathrm{Sb}$ that occurs as $\mu_{\mathrm{Sb}}$ decreases is isoelectronic, leaving the surface charge neutrality unchanged.

The III-Sb heterodimers undergo an electronic and structural relaxation similar to that observed on III-V (110) surfaces. An electron is transferred between the atoms, leaving the $\mathrm{Sb}$ with a filled lone pair and the $\mathrm{Al}$ with an empty lone pair. This electron transfer is accompanied by structural relaxation, where the $\mathrm{Al}$ relaxes down towards the plane defined by its three $\mathrm{Sb}$ neighbors, causing the two $\mathrm{Sb}$ neighbors in the layer below to move apart. The resulting structure accounts for the appearance of the heterodimers in the STM images: only the Sb filled lone pairs are observed in filled states, but the empty states are a more subtle blend from both the $\mathrm{Al}$ atoms and the Sb dimers.

Although transformation between the $\alpha$ and $\beta$ phases can occur via direct $\mathrm{Al} / \mathrm{Sb}$ substitution, the $\beta$-to- $\gamma$ and $\gamma$-to- $c(4 \times 4)$ transitions are slightly more complex. Strictly speaking, the $\gamma$ phase actually has a $(12 \times 3)$ conventional unit cell due to the peculiar alignment of the dimers from kink to kink. This symmetry probably arises because the $\gamma$ phase is structurally constrained between $\beta$ and $c(4 \times 4)$, giving $\gamma$ characteristics common to both. However, transforming from $\gamma$ to either phase requires significant surface rearrangement. Hence, it is possible that the narrow temperature range over which we observe $\gamma$ is due to a metastable extension of both the $\beta$ and $c(4 \times 4)$ phase regions into the actual region of $\gamma$ stability.

To our knowledge, III-V heterodimers like those integral to both the $\alpha$ and $\beta(4 \times 3)$ reconstructions have not been previously observed on a III-V device growth surface [22]. 


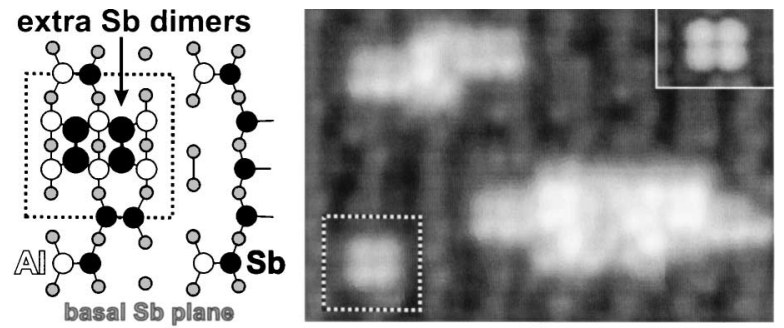

FIG. 3. Filled-state STM image of an AlSb(001)-(4 × 3) surface following deposition of about 0.2 ML of additional AlSb. A structural model for what appears to be the critical nucleus is shown on the left. A simulated image of this structure is inset on the upper right.

This structure is significant because the group III atom in the mixed dimer is spatially close to its bulk lattice site. Generally, the antimonides are distinct from other III-V's because they form (001) reconstructions with multiple $\mathrm{Sb}$ layers. As a consequence, the upper Sb layers must be displaced during epitaxial growth. However, because the $\mathrm{Al}$ atoms in the heterodimers are properly positioned for incorporation into the next layer during growth, they are natural sites for nucleation. Moreover, an $\mathrm{Al}$ atom deposited on the $\beta(4 \times 3)$ growth surface can incorporate directly into the lattice via creation of a mixed dimer with very little change in the surface energy. Such a growth mechanism would be fundamentally different than any observed for the other III-V surfaces.

We have preliminary evidence that these novel $(4 \times 3)$ reconstructions of the III-Sb(001) surfaces, in fact, play such a role during nucleation and growth. Following submonolayer homoepitaxy on AlSb and GaSb, small structures are observed that appear to be the critical nuclei for growth. As shown in Fig. 3, these small structures span two dimer rows and are about two dimers long. Our calculations show that the lowest energy configuration for these structures involves six $\mathrm{Al}$ atoms supporting two rotated $\mathrm{Sb}$ dimers. Although the exact formation process for these critical nuclei is not yet clear, the surrounding reconstruction likely plays a distinct role during subsequent growth. For example, as an island grows, $\mathrm{Al}$ can incorporate into the surface initially as heterodimers in [110]-neighboring $(4 \times 3)$ cells, which would allow additional $\mathrm{Sb}$ dimers to adsorb. As seen in Fig. 3, larger islands appear to be composed of multiple units of this structure, and when they are large enough, the secondlayer dimer row forms on top to make a (disordered) $(4 \times 3)$-reconstructed island. These results further demonstrate that a detailed understanding of surface structure is required to develop accurate atomistic models of film growth.

We thank B.R. Bennett, M.F. Gyure, F. Grosse, and J. Zinck for helpful discussions, and J. Furthmüller for generating the hydrogen pseudopotentials of fractional charges. This work was supported by the Office of Naval Research and DARPA under the Virtual Integrated Prototyping Initiative.

*Current address: HRL Laboratories, Malibu, CA.

Electronic address: wbc@hrl.com

†Electronic address: Lloyd.Whitman@nrl.navy.mil

[1] Z. Zhang and M. G. Lagally, Science 276, 377 (1997), and references therein.

[2] G. Klimeck, R. Lake, and D. K. Blanks, Phys. Rev. B 58, 7279 (1998).

[3] D. Z.-Y. Ting and T. C. McGill, J. Vac. Sci. Technol. B 14, 2790 (1996).

[4] Q.-K Xue, T. Hashizume, and T. Sakurai, Prog. Surf. Sci. 56, 1 (1997), and references therein.

[5] N. Esser et al., J. Vac. Sci. Technol. B 17, 1691 (1999).

[6] M. Itoh et al., Phys. Rev. Lett. 81, 633 (1998).

[7] P. Kratzer, C. G. Morgan, and M. Scheffler, Phys. Rev. B 59, 15246 (1999).

[8] B. V. Shanabrook et al., in Engineered Nanostructural Films and Materials, SPIE Proceedings Series Vol. 3790 (SPIE, Bellingham, WA, 1999), p. 13.

[9] C. F. McConville et al., Phys. Rev. B 50, 14965 (1994).

[10] L. J. Whitman et al., Phys. Rev. Lett. 79, 693 (1997).

[11] R. Ludeke, Phys. Rev. Lett. 39, 1042 (1977).

[12] J. R. Waterman, B. V. Shanabrook, and R. J. Wagner, J. Vac. Sci. Technol. B 10, 895 (1992).

[13] M. T. Sieger, T. Miller, and T.-C. Chiang, Phys. Rev. B 52, 8256 (1995).

[14] G. E. Franklin et al., Phys. Rev. B 41, 12619 (1990).

[15] P. M. Thibado et al., J. Cryst. Growth 175-176, 317 (1997).

[16] U. Resch-Esser et al., Phys. Rev. B 55, 15401 (1997).

[17] L. J. Whitman et al., J. Vac. Sci. Technol. B 14, 1870 (1996).

[18] B. Z. Nosho et al., J. Vac. Sci. Technol. B 17, 1786 (1999).

[19] G. Kresse and J. Hafner, Phys. Rev. B 47, R558 (1993); G. Kresse and J. Furthmüller, Phys. Rev. B 54, 11169 (1996).

[20] D. Vanderbilt, Phys. Rev. B 41, 7892 (1990).

[21] G. Kresse and J. Hafner, J. Phys. Condens. Matter 6, 8245 (1994).

[22] Structures incorporating mixed III-P dimers have been theoretically predicted for III-rich $\mathrm{GaP}$ and $\mathrm{InP}(001)$, and there is optical spectroscopy evidence of their occurrence, but there is no evidence they play any role in epitaxy. See Ref. [5]. 